\title{
Le nu couché dans la peinture de la Renaissance
}

\author{
Daniel Arasse
}

\section{(2) OpenEdition \\ Journals}

\section{Édition électronique}

URL : http://journals.openedition.org/shakespeare/1202

DOI : 10.4000/shakespeare.1202

ISSN : 2271-6424

Éditeur

Société Française Shakespeare

Édition imprimée

Date de publication : 1 novembre 1991

Pagination : 175-187

Référence électronique

Daniel Arasse, "Le nu couché dans la peinture de la Renaissance », Actes des congrès de la Société française Shakespeare [En ligne], 9 | 1991, mis en ligne le 01 janvier 2007, consulté le 01 mai 2019. URL : http://journals.openedition.org/shakespeare/1202 ; DOI : 10.4000/shakespeare.1202 


\section{SHAKESPEARE \\ ET LE CORPS A LA RENAISSANCE}

Société Française Shakespeare

Actes du Congrès 1990

sous la direction

de

M.T. JONES-DAVIES

PARIS

LES BELLES LETTRES

1991 
Tous droits de traduction, de reproduction et d'adaptation réservés pour tous les pays.

(C) 1991 Société d'édition Les Belles Lettres, 95 bd Raspail 75006 Paris

ISBN $2.25169117-\mathrm{X}$ 


\section{Le nu couché dans la peinture de la Renaissance}

Dans ce colloque consacré au corps à la Renaissance, j'interviens comme historien de la peinture. Le champ ainsi ouvert est vaste, de la théorie des proportions à l'anatomie et à cette nouvelle représentation du corps que constitue, en particulier, le De fabrica corporis humani de Vésale dont certaines planches ont été dessinées, faut-il le rappeler?, par Titien ... Pour ne pas être trop général, je voudrais n'envisager ici qu'un aspect de l'image du corps à la Renaissance : l'avènement en peinture du nu féminin, qui constitue aussi un événement considérable dans la culture de la Renaissance.

Et je me limiterai à tenter de comprendre l'importance et la signification historiques d'une seule cuvre: la Vénus d'Urbin, peinte en 1538 par Titien. Comprendre la Vénus d'Urbin, c'est-àdire comprendre comment, au sein de cette série de 'nus féminins couchés' qui traversent l'histoire de la peinture européenne, du $\mathrm{XVI}^{\mathrm{e}}$ siècle jusqu'à la fin du XIX ${ }^{\mathrm{e}}$ siècle, "irnage a pu constituer une nouveauté et continuer ensuite travailler l'histoire de la peinture au point que Manet y revient, la copie et s'en inspire directement pour réaliser, à son tour, une cuvre radicalement novatrice, Olympia.

Pour être limitée, cette approche de l'image du nu féminin à la Renaissance n'en est pas moins complexe.

Car la Vénus d'Urbin n'est pas la première œuvre de la série considérable de "nus féminins couchés" qui occupent pendant plus de trois siècles l'imaginaire érotique de la peinture européenne. Elle constitiue déjà une transformation d'une première œuvre, le véritable "chef d'œuvre" de la série : la Vénus de Dresde de Giorgione - conçue une trentaine d'années avant la toile du Titien et achevée par ce demier à la mort de Giorgione. Mais, dans sa conception générale, la peinture de Giorgione constitue elle-même une adaptation de modèles antérieurs, depuis un faux antique apparu dans la description d'une prétendue fontaine sculptée au bord du Danube (!) 
jusqu'à un antique véritable retrouvé à Rome sur la fin du XV siècle mais interprétée de maniète crronée (on y a vu une Didon mourante alors qu'il s'agissait d'une Aricane endormie). La Vérus de Dresde adapte ces sources diverses pour créer la première image peinte où le corps féminin constistue, dans sa nudité, J'objet principal et presque exclusif de l'attention; pourtant, il faut inmédiatement le souligner, ce que nous en voyons aujourd'hui th'est pas ce qu'en avait peint Giorgione : non seulement Titien a achevé louvre et, semble-t-il, ajoute en particulier les draperies qui isolent le corps de la prairie, mais la "Vénus" était accompagnée, sur la droite de l'cuvre, d'un petit Cupidon tenant un oiseau dont la trace est encore repérable par radiographie. Ainsi, non seulement l'idée originelle de Giorgione a tét, très tôt, transformée par Titien, mais cette transformation elle-même a été, à son tour, modifiée par I'histoire et la succession de ses interventions.

Ces remarques n'auraien quine valeur documentaire si on n'en concluait pas l'évidence : le prototype du nu féminin couché de la peinture européenne est insaisissable. La Vénus de Dresde n'en touche pas moins loin et profond, aussi bien en ce qui concerne les mécanismes psychiques du spectateur dans sa "réception" de l'curvre que les fins de la peinture elle-même, dans sa production et la mise en auvre de ses moyens:

- 'Réception' de l'cuvre : il n'est que de lire les lignes que Kenneth Clark consacre à la toile de Giorgione pour percevoir la complexité des enjeux de ce que le tableau propose au regard: «C'est parce que Giorgione a su découvrir soudain la forme et la couleur des désirs qui flottaient de façon à demi consciente dans l'esprit de ses contemporains que son cuvre nous est parvenue inextricablement confondue avec celle d'autres artistes, Giorgione n'eut pas plus tôt découvert le mot de passe que tous purent franchir la même porte et un ou deux d'entre eux le dépassèrent probablement.» 'Formes et couleurs de désirs flottants', 'mot de passe', 'porte ouverte et franchie' : l'absurdité très personnelie des métaphores de Kenneth Clark est par elle-même révélatrice : la Vénus de Dresde est une image "efficace".

- Cette efficacité touche par ailleurs aux fins mêmes de la peinture. Car Giorgione, le "gros Georges" si l'on s'en tient à la lettre de son surnom, est aussi l'auteur supposé d'un tableau disparu dont l'histoire posthume est hautement significative. Il aurait peint un tableau admirable, représentant Saint Georges près d'une source et permettant de voir la figure sous tous ses aspects grâce à 
son double reflet dans l'eau d'une part et, d'autre part, de dos, dans son bouclier posé contre un arbre. Tel ce Narcisse qu'Alberti met, en 1435 , a la source de la peinture (et mieux encore que lui), Giorgione apparaît ainsi comme l'inventeur d'une peinture qui fait tout voir, où il se peint lui-même (Georges), tout en s'y perdant. Ce tableau n'a sans doute jamais existé et il s'agit plutôt d'une référence inventée pour les besoins d'une juste cause : permettre aux contemporains de condenser en une seule image, même inexistante, le propre de la peinture giorgionesque. C'est du moins ce que le destin littéraire de ce tableau "perdu" conduit à supposer: deux ans après son apparition, en 1548, dans le dialogue vénitien de Paolo Pino, le "Saint Georges de Giorgione" réapparaît sous la plume du Florentin Giorgio Vasari; il y connaît la première phase de sa métamorphose (narcissique?) en devenant un "nu à la source"; la métamorphose est accomplie quelques années plus tard quand, dans son Idea del Tempio della Pittura, le Lombard Lomazzo en fait, finalement; «la pittura ignuda nella fonte», la peinture nue à la source, la Peinture elle-même qui se reflète dans ses propres œuvres. Cette transformation progressive d'un tableau inexistant, où Giorgione en saint Georges avec son armure devient la Peinture même en femme nue, est fascinante : dans la représentation du corps nu, dont l'école vénitienne est en effet la grande spécialiste, c'est le corps même de la peinture qui est en jeu ...

Il est temps, après ce détour initial, de revenir à la Vénus d'Urbin, avec une triple question :

1. Pourquoi et en quoi cette toile constitue-t-elle une nouveauté? à quelle "opération" Titien s'est-il livré par rapport à son modèle giorgionesque et aux divers modèles apparus entre 1508 (date approximative de l'Guuvre de Giorgione) et 1538 ?

2. Qu'y a-t-il, dans cette cuvre et cette opération, qui explique l'impact de la Vénus d'Urbin, et le fait qu'elle est, plus de trois siècles plus tard, le modèle direct de cette Olympia scandaleuse?

3. En quoi ce travail sur la peinture du nu féminin participe-til du renouvellement "Renaissance" de l'image du corps?

La première nouveauté, manifeste, qu'introduit Titien dans le traitement du nu féminin consiste à placer celui-ci dans un intérieur et non plus dans la nature. Par là-même, il exclut la réception de l'image comme image de nymphe.

Par ailleurs, en évacuant toute détermination narrative précise et, mises à part les deux servantes de l'arrière-plan, toute iconographie secondaire, il rend problématique, pour reprendre la termi- 
nologie d'Erwin Panofsky, lidentification d'un 'thème' particulier dans le 'motif' de la femme nue couchée. Il est à noter d'ailleurs que la dénomination de "Vénus" est tardive : dans la correspondance échangée entre le commanditaire de l'cuvre et l'artiste, la toile est désigné, de manière significative, comme "la femme

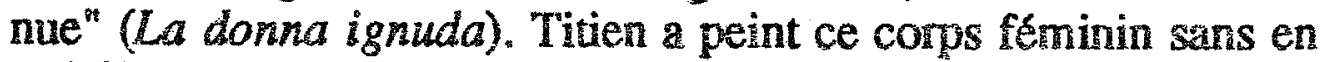
spécifier d'articulation narrative, mythologique on allegorique. Cette décision explique la variété remarquable des interprétations proposées pour définir l'identitê du personnage féminin : depuis lidée d'une Vénus nue (c'est-à-dire dans le contexte néoplatonicien dominant de l'époque une "Vênus céleste" et spirituelle) jusqu'à l'image d'une courtisane, une prostituće (dont on sait que Venise étaii abondamment et brillamment pourvue), en passant par l'allusion à un "portrait" idéalisé (dans le cas où le tableau serait un "tableau de mariage", comme l'époque en produit souvent) ou par le charme plus simple d'une "pin up", comme le propose le très sérieux Charles Hope, avec toute l'autorité que donne le prestige du Warburg Institute ...

Que l'indétermination iconographique fasse partie de lintention du tableau est confirmé par un détail auquel les interprètes de l'œuvre n'ont pas assez porté attention, trop pressés de se rassurer par une claire "lecture" des images.

Il est impossible en effet de déterminer comment s'articuleraient, dans une architecture supposée réelle, les deux zones du tableau (premier plan avec le lit / arrière-plan avec la salle et les servantes): non seulement le pavement de la salle s'arrête aui "bord" du lit sans que la nature de ce bord soit identifiable, mais la très forte verticale qui, au centre du tableau, marque la séparation entre ces deux zones est elle aussi, décidément, non identifiable, au niveau même de ce que Panofsky appelle la «description préiconographique» : impossible d'affirmer qu'il s'agit d'un rideau, d'un mur ou d'un meuble : un rideau vert est clairement "lisible" dans la partie supérieure gauche de la toile, mais la grande zone sombre ne porte aucune définition interne qui permettrait de la "lire" comme tel ou tel objet. Il faut d'ailleurs souligner que, si l'on veut reconnaître dans ces deux "zones" du tableau deux parties d'une même pièce de palais ou deux pièces dans un même palais, il devient impossible d'en concevoir le rapport de manière vraisemblable : le lit de "Vénus" serait en effet alors posé à même le sol (situation inimaginable); ou il faudrait supposer une 
dénivellation entre les deux "pièces" dont aucune trace n'est laissée dans l'image ...

Ces remarques ont un seul intérêt : elles conduisent à la conclusion que les deux zones du tableau ne sont pas à concevoir comme deux "endroits" d'un palais fictif, mais bien comme deux "lieux" figuratifs dont le rapport se construit et s'interprète à lintérieur de la toile elle-meme. Car, de ce point de vue, la relation dialectique qu'entretiennent les deux lieux est évidente, manifeste : nudité/habillement, couché/debout, courbes/droites, horizontales/ verticales, etc. : les deux parties du tableau se définissent réciproquement à l'intérieur même du tableau et, de manière très neuve, presque radicale, Titien construit une image qui élabore son sens à partir de sa propre articulation interne, quelle que soit par ailleurs la justification iconographique que l'on veuille et que l'on puisse donner à la toile.

Pour dégager le sens que ce travail de Titien donne au tableau, il convient donc de considérer avec précision sa construction interne.

On est frappé, de ce point de vue, par l'importance et la clarté de la construction perspective de l'arrière-plan. On en est frappé car Titien ne s'intéresse guère, d'habitude, à la construction "légitime" de ses espaces picturaux, et ce type de problématique est d'ailleurs historiquement dépassé en 1538. Il est vrai cependant aussi qu'à deux reprises au moins, Titien a déjà montré qu'il était capable, dans une cuvre radicalement novatrice, de recourir à ce type de pratique (je pense ici à l'Annonciation qu'il peint à Trévise en 1520 et où, tout en faisant entrer l'ange par la droite et le fond de l'image, il l'installe sur un magnifique pavement en perspective au damier rigoureusement construit; je pense aussi au Miracle du nouveau-né peint à la Scuola de saint Antoine à Padoue vers 1511 et à la brutale articulation verticale qui, au centre de l'image, sépare le mur et le paysage).

Titien a en effet rigoureusement construit la perspective du sol représenté à l'arrière plan : ses lignes de fuite convergent vers une zone située au centre de l'image, pratiquement sur la ligne verticale qui sépare les deux "lieux" du tableau et à la hauteur exacte de l'œil gauche de "Vénus" (il suppose ainsi un spectateur dont l'œil est situé au centre de l'image, à la hauteur du visage de "Vénus"); par ailleurs le "point de distance" (qui indique la distance supposée du spectateur au plan idéal du tableau), le point de distance est situé sur le bord droit du tableau. Cette construction, 
encore une fois étonnamment rigoureuse et, donc, très sciemment calculée, implique une conséquence capitale : Titien a voulu situer le spectateur du corps féminin au plus près de ce corps et à sa hauteur, comme à genoux devant ce corps, puisque l'ensemble de l'image semble situer le lit au niveau du sol.

Cette observation ouvre sans doute l'interprétation de l'œuvre. Celle-ci comporte en effet, en son sein même, une figure qui répète cette position; il s'agit bien sûr de la "servante" agenouillée à l'arrière-plan devant le coffre, au plus près de son ouverture. Anecdotiquement ou iconographiquement incertain, le rôle que joue cette figure au sein du tableau est par contre décisif tant du point de vue de sa structure que de son contenu.

Les coffres situés à l'arrière-plan sont en effet en général considérés comme une allusion à ces 'coffres de mariage' (cassoni) qui accompagnaient la jeune épousée et dans lesquels pouvait également se trouver une part de sa dot et de son "trousseau"; or c'est à l'intérieur de ces coffres de mariage, très exactement au revers de leur couvercle, qu'ont été peints les premiers "nus féminins couchés" de la peinture européenne, comme le notait déjà Kenneth Clark à propos de la Vénus de Dresde.

Mais il en tirait alors une conclusion insuffisante car il y voyait seulement les 'véritables ancêtres' de l'œuvre de Giorgione. Or c'est bien davantage par rapport à la toile de Titien que ces nus couchés au revers des couvercles de coffre jouent leur rôle d'ancêtres'. Ils le jouent même de façon très précise : l'opération (proprement génial) à laquelle s'est livré Titien a consisté à 'faire sortir' le nu féminin couché du coffre où il était enfermé pour le mettre et le projeter au premier plan de la peinture elle-même. Cette opération est suggérée par la figure de la servante agenouillée à l'arrière-plan, dans la même situation que le spectateur par rapport au corps de "Vénus"; elle est aussi marquée par l'indétermination de la zone sombre qui sert de fond à la partie supérieure du corps de "Vénus"; il ne s'agit pas de dire que cette zone sombre "représente" le revers du couvercle du coffre, mais plutôt que son indétermination iconographique "figure" l'opération même de déplacement opérée par Titien.

Celle-ci est d'une portée considérable.

En projetant au premier plan de la peinture une image traditionnellement située à l'intérieur d'un coffre de mariage, Titien bouleverse en effet le statut qui était celui de cette image, dont la vision était réservée à celui ou celle qui était susceptible d'ouvrir le 
coffre, une vision "privée" donc et, plus encore, "intime". La structure du tableau prend, dans ce contexte, un sens remarquablement précis : du premier plan jusqu'à l'arrière-plan le plus lointain (la nature entrevue à travers la fenêtre), trois "zones" ou trois "sphères" se succèdent: sphère "intime" de la nudité dévoilée, sphère "privée" de la salle avec ses coffres et ses vêtements, sphère "publique" de la nature. Cette articulation en profondeur suscite un effet de sens remarquable; dans cet espace de jouissance, à la fois privé et public, qu'est celui du collectionneur, Titien rend visible une intimité. Il "pro-stitue" ainsi, si l'on peut dire, cette intimité en la situant sur le devant de la peinture. Cela contribue sans doute aussi à expliquer les interprétations de "Vénus" comme "courtisane" ou "pin up"; mais ces interprétations sont manifestement réductrices. Pour situer la portée proprement historique de l'invention' de Titien, il convient plutôt de constater que sa peinture nous fait voir, nous admet à la contemplation d'une beauté nue dont les pratiques sociales de l'époque nous excluraient normalement; la Vénus d'Urbin nous «fait voir ce que nous ne verrions jamais» et la formule est à entendre en un double sens :

- La peinture de Titien est, plus qu'aucune autre, capable de peindre la beauté du corps féminin; l'opération du peintre est aussi à la gloire de son œuvre.

- La peinture de Titien nous fait voir un 'état du corps' dont aucun équivalent n'existe dans la nature même ou, pour le dire comme le contemporain Sperone Speroni, les peintures de Titien sont «le paradis de nos corps». Formule à prendre presque au pied de la lettre; ce que nous fait voir la Vénus d'Urbin, c'est un "corps glorieux", c'est la gloire d'un corps ressuscité, suscité par la peinture même. En ce sens, la Vénus d'Urbin est inséparable des autres "corps glorieux" qui ont tant fait pour la gloire du peintre : Danaé, Europe enlevée par Jupiter, Diane, Madeleine même ... Elle fait partie de cette série glorieuse; mais elle en fait partie comme marginalement, et avec une force de fascination peut-être plus grande encore dans la mesure où cette gloire dévoilée est aussi celle d'une intimité au bord d'être partagée, exposée à la caresse d'un regard rapproché, pleinement visible hors de toute justification mythologique, invisible hors de ce coup de force qui a arraché la beauté du coffre où l'enfermait la censure du regard.

Cette interprétation permet de saisir comment le peintre a travaillé avec et sur un matériau qui lui était offert par la tradition contemporaine; mais elle n'explique pas encore comment, plus que 
la Vénus de Dresde, c'est celle dUrbin qui a pu devenir la matrice de cet autre grand nu féminin mythique de la peinture européenne, l'Olympia.

Il faut revenir à une comparaison serrée entre les deux œuvres, sans oublier que la première a été achevée par l'auteur de la seconde, qu'il la connaissait donc avec une intensité et une intimité exceptionnelles. Le regard doit, ici, scruter au plus près les configurations de la figure.

On a déjà dit l'importance du "passage à l'intérieur" d'une cuvre à l'autre. On s'en tiendra ici au travail sur la configuration du corps même, envisagé à travers le rapport nouveau que ce travail instaure entre la peinture et celui qui la regarde.

Première remarque, bien connue; la Vénus de Giorgione dort, celle de Titien est éveillée et me regarde. De l'une à l'autre, c'est tout le thème de la "Vénus pudica" qui est mis en jeu et avec lequel joue le peintre dans la ressemblance même d'une figure à l'autre. Car, si la première est, comme on dit, "inconsciente" du regard que je pose sur elle, la seconde ne saurait lêtre. Le rapport à l'intimité dévoilée en est évidemment bouleversé.

Deuxième remarque, étroitement liée à la première : la pose du bras droit de la figure est changée. La Vénus de Dresde a le bras replié derrière la tête, dégageant ainsi la vue de son aisselle, zone habituellement cachée du corps. La Vénus d'Urbin a baissé le bras sur le coussin; elle me cache cette zone intime et me la voile, de surcroit, par une abondante chevelure blonde dénouée.

A ce point, je remarque que la chevelure de Vénus a changé de couleur de cheveux : brune chez Giorgione (cas rare pour une Vénus vénitienne - et, d'ailleurs, ses cheveux sont à peine visibles, passés derrière la tête), elle est devenue plus normalement d'une magnifique blondeur chez Titien. Par ailleurs, et je touche là sans doute à un point essentiel du système de transformation qui rapporte le second tableau au premier, si la première Vénus a le bras soulevé, je n'ai "rien" à voir en ce lieu qu'elle me montre (inconsciemment); au contraire, Titien me cache l'aisselle mais il me montre une abondante chevelure à la place de ce repli intime, épilé chez Giorgione. Je constate alors que ce processus de déplacement se répète pour l'autre bras de "Vénus" : la pose en est pratiquement identique (évoquant encore le geste antique de la "Vénus pudica"); mais chez Giorgione cette main pudique me fait voir un pubis également épilé, alors que Titien a placé, là, une ombre profonde. Anatomiquement injustifiée, elle ne se justifie 
donc que par une recherche tactique d'effet : cette ombre m'indique que je ne vois pas ce que je pourrais (mais ne saurais, comme dira Tartufe) voir; mais elle fait mieux : elle me suggère aussi cette chose que je ne vois pas.

On aura compris que, d'un tableau à l'autre, le jeu des mains de la figure est devenu plus séducteur, proprement érotique selon la pratique ancestralement connue du «je te cache ce que je te montre, je te montre ce que je te cache» - et d'ailleurs, cette pratique n'est pas sans rapport avec la situation ancienne de cette femme nue, peinte au revers du couvercle d'un coffre et visible donc seulement de façon intermittente ... Mais il est également intéressant de constater que cette tactique érotique opère au registre de la pilosité intime. Giorgione l'avait simplement exclue de la représentation; au contraire, Titien se livre à une double opération de suggestion: suggestion par détournement (l'ombre du pubis) et suggestion par déplacement (les cheveux blonds à l'emplacement de l'aisselle cachée). Le travail du peintre opère ainsi sur un élément du corps aux connotations multiples: les cheveux et les poils dont les textes contemporains confirment la valeur particulièrement érotisée; on sait par exemple que, pour les femmes mariées, le cođe social et religieux en régissait avec précision la "visibilité publique" - et on ne saurait oublier non plus l'impact particulièrement somptueux que Titien donne aux cheveux de ses Saintes Madeleine ... Titien joue en fait très consciemment avec cette substitution de la chevelure au poils intimes et il élabore ainsi une des images les plus anciennes à être aussi délibérément érotique, tout en situant cet érotisme au sein d'une image dont la structure elle-même travaille la limite entre sphère intime et sphère privée.

Le caractère systématique de cette transformation est confirmé par la réélaboration de la main gauche; chez Titien, le pouce a rejoint l'index; il constitue ainsi un interstice ombreux là où Giorgione avait séparé les doigts, empêchant toute suggestion "impudique".

L'aspect méticuleux de la description qui précède, et du système de transsformation qu'elle suppose, ne doit pas surprendre : non seulement Titien avait achevé la "Vénus" de Giorgione (il la connaissait donc "de près"), mais, on l'a vu, la construction perspective de son tableau suppose son spectateur au plus près de la surface. L'effet peut-être le plus important de son travail tient à la présence inhabituelle de ce nu féminin; le regard de la "femme nue", de la donna ignuda, impatiemment réclamée au peintre par 
son commanditaire, est ouvert sur le spectateur; par là, la figure annonce moins son "impudeur iconographique" qu'elle ne manifeste la conscience d'être vue, offerte au regard, impliquant par cet œil qui porte son coup le spectateur dans sa propre présence.

Titien instaure ainsi un rapport nouveau entre la peinture et celui qui la regarde. Le regard rapproché et intime n'est plus celui qu'impliquait naguère le rapport de dévotion et l'affectum devotionis que devait susciter, par exemple, la contemplation rapprochée des plaies du Christ jusqu'à pousser le dévot à en embrasser la représentation. Il n'est pas question pour le propriétaire de la Vénus d'Urbin d'en embrasser la surface peinte, mais le regard rapproché que le tableau suscite vise bien une jouissance, celle que suscite la présence imaginaire d'un corps fictif.

C'est sans doute dans cette co-présence suggérée de la peinture et de son spectateur que réside l'extraordinaire capacité de fascination qu'a exercée la Vénus d'Urbin, "corps glorieux" intimement dévoilé, érotiqument montré, dont Manet donnera la version moderne et đésacralisante.

Je conclurai rapidement en tentant de répondre à la troisième des questions que je posai au début : en quoi ce travail de la peinture sur le nu féminin participe-t-il du renouvellement "Renaissance" de limage du corps?

La question est difficile et, pour y répondre, il vaut mieux sans doute partir de constatations simples.

En 1538, Titien a environ 50 ans et il connait une gloire sans égale : c'est l'époque (1538-39) où il peint en même temps les portraits du roi de France François Ier et du Saint Empereur Charles-Quint; il est le "nouvel Apelle" des nouveaux Alexandre. Mais 1538 est aussi l'année d'une grave crise dans son métier de peintre officiel de la République Sérénissime : la charge du Fondaco dei Tedeschi, qui revient à reconnaissance de "Peintre d'Etat" lui est alors retirée pour être attribuée à son rival du Frioul, le tumultueux Pordenone. Pordenone meurt en 1539 et Titien retrouve sa charge. Mais l'année 1538 n'en a pas moins été une année charnière dans une carrière déjà longue: Titien a vu osciller son prestige, à la limite entre domaine public et domaine privé de l'exercice de son pouvoir.

Or la Vénus d'Urbin, commande très privée de Guidobaldo della l.overe, est son premier "nu féminin couché"; il reviendra ensuite abondamment sur le motif, à partir de 1545 et jusqu'aux 
années 1570, avec les multiples versions de Danaé et des Vénus au musicien. Progressivement, sa peinture du corps féminin devient un véritable "corps à corps" avec la peinture elle-même; c'est cette dernière manière, ultima maniera, magnifiquement décrite par Boschini : «Il recouvrait ces quintessences de couches superposées de chair vivante, jusqu'à ce que seul le souffle semblât leur manquer ... Il donnait le fini aux dernières retouches en frottant plusieurs fois du doigt les passages des parties claires aux demiteintes ... D'autre fois, toujours avec ses doigts, il posait une tache de noir dans un coin, ou bien d'une pointe de rouge il rehaussait une carnation, comme d'une goutte de sang ...»

Titien exalte alors le corps pictural de la peinture. Ce n'est pas encore le cas en 1538 et ce rapprochement met en lumière le caractère très conçu de la Vénus d'Urbin. La strscture géométrique évidente de la surface picturale manifeste qu'un concept, un concetto comme on disait alors, travaille l'image, concetto dont la mise au point en peinture prépare l'évolution du peintre vers son ultima maniera.

On a vu comment la conception de l'œuvre s'élaborait à partir de la manipulaton d'un matériel traditionnel. On ne peut sans doute en rester là car lidée même de cette manipulation dépendait aussi du contexte théorique et philosophique, du concept qui en rendait possible l'émergence. C'est ce dernier qu'il faut maintenant envisager.

De ce point de vue, il convient de partir de l'arrière-plan iconographique de l'Euvre. C'est lui qui permet en effet de situer l'horizon d'attente' à laquelle la toile répond, qu'elle comble même tout en y introduisant l'écart' qui définit aussi, historiquement, le chef-d'cuvre.

Or, à Venise et dans l'œuvre de Titien en particulier, la dialectique signalée plus haut entre nudité (affichée au premier plan) et habillement (impliqué à l'arrière-plan) est idéologiquement lié au thème des "deux Vénus" néoplatoniciennes. Magistralement reconnues jadis par Panofsky dans L'Amour sacré et l'amour profane peint par Titien une vingtaine d'années avant la Vénus d'Urbin, Vénus nue est la Vénus céleste, spirituelle; Vénus habillée est la Vénus terrestre, non pas Vénus vénale mais incarnation du principe de beauté et d'harmonie cosmique reflété dans les beautés visibles.

Cependant, si L'Amour sacré et l'amour profane affichait cette dualité en la représentant à travers les figures jumelles des deux 
Vénus, la toile peinte en 1538 rend, on l'a dit, le thème beaucoup plus problématique en le condensant en une seule figure, et en évacuant de la représentation tout ce qui aurait permis l'identification sûre d'une métaphysique néoplatonicienne.

Par cette incertitude où, dans sa réception de l'image, le spectateur est laissé quant à la finalité de la représentation et à son "interprétation secondaire", le peintre exalte certes sa propre peinture, manifestation aussi évidente qu'incertaine d'une beauté "glorieuse".

Mais il fait sans doute plus encore.

Libre de son interprétation, le spectateur est mis en situation de choisir la dimension spirituelle et mentale qu'il accordera à l'image : «Qua sù o qua giù». Par là même, le spectateur de la Vénus d'Urbin est dans la situation que la métaphysique néoplatonicienne donne à l'homme dans son cosmos : artiste de luimême et maître de sa destinée, il est susceptible de faire l'ange comme la bête, seule créature investie de la responsabilité d'un tel choix.

On aura reconnu la paraphrase d'une page célèbre du Discours de Pic de la Mirandole sur la "dignité de l'homme". Il ne s'agit pas de dire que ce Discours inspire le travail de Titien, mais plutôt de le reconnaître un possible arrière-plan banalisé du "flou iconographique" dans lequel est présentée la donna ignuda : beauté à la fois céleste et érotiquement intime, douée d'une présence énigmatique qu'il tient au spectatuer d'actualiser dans sa contemplation, selon tel ou tel mode.

C'est sans doute finalement cette présence de l'image et sa coprésence au spectateur qui en définit peut-être le mieux l'esprit "Renaissance".

L'effet de "présence" construit par Titien dans son tableau n'est pas seulement celui d'une présence physique : c'est aussi celui d'un présent de la représentation. On sait que ce présent de la représentaton est une des grandes recherches de la peinture Renaissance; on sait que Venise y travaille par ce "je ne sais quoi" de vivant que donnent le coloris et sa magie; on sait aussi que, dès le texte fondateur d'Alberti sur la peinture, écrit presque exactement un siècle avant que la Vénus d'Urbin ne voie le jour, la «force presque divine» de la peinture tenait à sa capacité de «rendre l'absent présent» et le mort «vivant» et, une page plus loin, Alberti affirmait que c'était Narcisse même que l'on devait considérer comme l'«inventeur de la peinture» ... Narcisse florentin dont la 
version vénitienne prendra la figure d'un Saint Georges à la fontaine métamorphosé en peinture nue ...

Que fait d'autre, en définitive, la Vénus d'Urbin sinon offrir à son spectateur le miroir de son désir, insaisissable en sa source et irreconnaissable en son «être-là»?

Son "corps glorieux" pourrait bien être le tout premier grand fétiche d'un Eros moderne.

Daniel ARASSE

Université de Paris I 